\title{
La opinión de los españoles ante las nuevas biotecnologías
}

\section{Juan Antonio Taguenca Belmonte}

Universidad Autónoma del Estado de Hidalgo. Instituto de Ciencias Sociales y Humanidades Área Académica de Ciencias Políticas y Administración Pública juantaguenca@yahooo.com.mx

\section{Resumen}

Las nuevas biotecnologías representan una gran esperanza para el ser humano. Sin embargo, la gente tiene una percepción respecto a ellas ambivalente, donde el bien y el mal que suponen están juntos. El presente artículo trata de esta ambivalencia en España. Para ello, he analizado cuatro estudios de opinión con diferentes metodologías: grupos de discusión, entrevistas y encuestas. El trabajo de análisis realizado permite hacer una evaluación comprehensiva del conocimiento, la información, el interés, la aceptación, la percepción, la demanda de control, el conocimiento de la normativa, la participación social reclamada, la percepción del riesgo, etc. Todos ellos aspectos muy importantes para legitimar las nuevas biotecnologías, y para que ellas tengan la aceptación del público.

Palabras clave: nuevas biotecnologías, opinión pública, España, ambivalencia.

\section{Abstract. The Spanish opinion in front of the new biotechnologies}

The new biotechnologies represent a great hope for the human being, although people have a perception respect they ambivalent, where the good and the bad that suppose are join. The present article treats this ambivalence in the Spain people. For that I have analysed four public opinion studies with different methodologies: discussion groups, interviews, and inquiries. The analysis work realized permit do a comprehensive evaluation for the knowledge, information, interest, acceptation, perception, control demand, normative knowledge, social participation claims, risk perception, etc. All these items are very important for legitimate the new biotechnologies, and for that they have the public acceptation.

Key words: new biotechnologies, public opinion, Spain, ambivalence.

\section{Sumario}

1. Introducción

2. Dificultades de definición
3. La opinión de los españoles ante las nuevas biotecnologías

Referencias bibliográficas 


\section{Introducción}

Las nuevas biotecnologías constituyen una de las mayores esperanzas, sino la mayor, en ámbitos de relevancia socioeconómica tan importantes como la agricultura, la ganadería, la producción de alimentos, la química fina, la salud y la farmaquímica, la minería, la producción de materias orgánicas a granel y el medio ambiente. Tiene aplicaciones en esos ámbitos de tanta incidencia como, por ejemplo: obtención de variedades de plantas tolerantes a condiciones ambientales negativas, más productivas y resistentes a enfermedades, fijación de nitrógeno o captación de elementos nutritivos, pesticidas microbianos, protección de inóculos, diagnóstico, prevención y control de enfermedades animales, nutrición y crecimiento animal; aditivos para la industria alimenticia, mejora de actividades biotecnológicas tradicionales, obtención de proteínas en gran cantidad a través de productos microbianos, producción a gran escala de enzimas; obtención de aminoácidos, vitaminas, polímeros, lípidos complejos, sustancias aromáticas; producción de productos farmacéuticos, antibióticos y vacunas, diagnóstico y tratamiento de enfermedades; recuperación de hidrocarburos; para usos diversos de la biomasa, y también se utilizan para el tratamiento de aguas, el tratamiento de desechos tóxicos y el control de metales pesados ${ }^{1}$.

Estas prometedoras aplicaciones de las nuevas tecnologías de la vida constituyen la base de su aceptación: son percibidas como positivas para el ser humano y el medio ambiente. Sin embargo, su percepción también da lugar a temor y oposición, lo que depende de qué sujeto, forma aplicativa e interés se intuya detrás de la tecnología de la vida considerada. Y es que el ser humano se enfrenta, por primera vez en su historia, a la posibilidad de crear nueva vida basada en la recombinación de la existente; lo que despierta recelos y temores de que la naturaleza pueda ser transformada profundamente (sólo atendiendo a consideraciones económicas y sin tener en cuenta los riesgos que dichos cambios suponen $)^{2}$; y sobre todo que el hombre pueda ser tratado más como un medio que como un fin. Recordemos, al respecto, la máxima kantiana: «actúa de tal manera que trates a la humanidad, tanto en tu persona como en la persona de cualquier otro, siempre como un fin al mismo tiempo y nunca como un medio» (Bilbeny, 1992: 72$)^{3}$. No podemos olvidar tampoco que tanto las aplicaciones de estas nuevas tecnologías de la vida como sus bases científi-

1. Esta lista de las aplicaciones de la biotecnología está extraída del cuadro que aparece en Solleiro (1990: 109).

2. M. Redclift y G. Woodgate señalan que: «In the process of coevolutionary change [el de la naturaleza y el de la sociedad], society has assumed more and more of the functions traditionally undertaken by nature [...] Aspects of 'nature have been refashioned and converted into industrial processes, under scientific control. This is particulary evident in the new biotechnology industries, and genetic engineering» (Redclift y Woodgate, 1997: 59). Esta transformación de la naturaleza en proceso industrial es la que despierta los recelos y los temores señalados.

3. Extraído de KanT, I. (1968). Grundlegung, Zur Metaphisik der Sitten. Kants Werke, Akademie Textausgabe. Berlín: De Gruyter, p. 429. 
cas son: "a big business and recombinant DNA research is no exception» (Krimsky, 1992: 275-276). Es esta dimensión de negocio industrial la que genera la ambivalencia de aceptación, al tiempo que de temor y rechazo, de las nuevas biotecnologías por parte de los ciudadanos.

En cuanto a las hipótesis que sustentan el presente artículo, y que tienen su correspondencia en la situación de ambivalencia resaltada en el párrafo anterior, son las siguientes:

\section{Primera:}

Las nuevas biotecnologías son aceptadas por los españoles, pero no es una aceptación incuestionada, pues la misma está matizada por los usos que se hagan de ellas. En definitiva, no todo uso de la biotecnología tiene el mismo grado de aceptación, e incluso existen aplicaciones que están fuertemente cuestionadas y rechazadas desde el lado de la legitimación otorgada por la opinión pública. En definitiva, mi hipótesis es que los españoles perciben las nuevas biotecnologías de forma ambivalente: sus beneficios y riesgos potenciales son percibidos, lo que produce al tiempo aceptación y rechazo de estas nuevas tecnologías de la vida.

Sostengo que lo dicho aquí como hipótesis para los españoles es válido para otros países en mayor o menor grado, con lo que las nuevas biotecnologías son diferenciadas en sus aplicaciones y en sus posibles beneficios y riesgos. Aunque aquí hay que distinguir claramente de qué desarrollos y aplicaciones se trata, pues existen diferencias por países.

\section{Segunda:}

Los españoles tienen una opinión pragmática ante las nuevas biotecnologías. Esto es, apoyan a las que perciben beneficiosas, sobre todo a aquéllas destinadas a la salud humana; se muestran contrarios a las aplicaciones que perciben como riesgos inaceptables: transformaciones genéticas de los seres humanos, sobre todo; y, en todo caso, son favorables a controlar y regular las aplicaciones biotecnológicas, en aras de disminuir los riesgos potenciales que de ellas derivan. En esto coinciden con la opinión de los ciudadanos de otros países, como mostraré en este artículo.

\section{Tercera:}

La tercera hipótesis es que la actitud de los españoles ante las nuevas biotecnologías es pasiva en cuanto a su participación en los debates y las políticas que sobre ellas se han realizado; pero también en cuanto a la adquisición del conocimiento mínimo necesario para una participación racional en los mismos, y ello a pesar del interés mostrado.

Por otro lado, la actitud es activa en cuanto a la reclamación de una mayor participación en estos debates y políticas; siendo los factores de regulación y 
control, tanto en el plano de la investigación como en los de desarrollo y aplicaciones, los más reclamados desde el punto de vista de reclamación participativa, lo cual no se traduce en conocimiento sobre las legislaciones de regulación y control existentes de estas nuevas tecnologías de la vida. En definitiva: la reclamación participativa no produjo participación efectiva, y el interés mostrado en este tema no tuvo su correlato en conocimiento fundamentado sobre el mismo.

Aquí hay que diferenciar entre distintos aspectos, pues si bien el interés y el control reclamado, a través de la normativa de las nuevas biotecnologías, son comunes en todas las ciudadanías de las naciones que mencionaremos, los niveles de información, conocimiento y debate alcanzados por éstas en los distintos países son distintos. Lo que no supone que las actitudes observadas en todos ellos dejen de ser ambivalentes, sino que se trata de ambivalencias distintas que hay que matizar.

\section{Cuarta:}

España, al igual que otros países, en la década de los noventa, estaba todavía en las primeras fases de formación de actitudes respecto a las nuevas biotecnologías. Éstas no se encontraban fijadas todavía en la mayor parte de la población.

Respecto a los objetivos del artículo, que tiene un alcance descriptivo y sintético, son los siguientes:

\section{Primero:}

Dar cuenta de la percepción, la opinión y la actitud de los españoles ante las nuevas biotecnologías en la década de los noventa. Para ello utilizo las hipótesis referidas en los párrafos anteriores y las contrasto con los resultados empíricos obtenidos en varias investigaciones sobre este tema, tanto cuantitativas como cualitativas, realizadas en nuestro país y en el marco de la Unión Europea.

\section{Segundo:}

Comparar la percepción, la opinión y la actitud de los españoles sobre las nuevas tecnologías de la vida con las que tienen los habitantes de otros países (dimensión geográfica), lo cual es interesante desde un doble punto de vista: da cuenta de la similitud y la disimilitud existente en nuestro país con otros países en cuanto a la percepción, la opinión y la actitud, sobre distintos desarrollos y aplicaciones biotecnológicas, y sitúa la verdadera dimensión del debate y la participación ciudadana existente sobre estas tecnologías, tanto en España como en otros estados. 


\section{Dificultades de definición}

La «opinión pública» es un concepto que resulta difícil definir desde las ciencias sociales. Al respecto, la sociología no representa una excepción. Autores como J. Habermas han señalado que: «se abren fundamentalmente dos caminos para definir el concepto de opinión pública. Uno de ellos retrotrae a posiciones liberales; el liberalismo quiso salvar la comunicación —en el ambiente de una publicidad desintegrada - de un círculo interno de representantes capaces de publicidad y formadores; el liberalismo quiso salvar un público raciocinante en el ambiente del público meramente aclamativo. [...] El otro camino lleva a un concepto de opinión pública que prescinde por completo de criterios materiales tales como racionalidad y "representación" y se limita a criterios institucionales» (Habermas, 1981: 263). Otro autor, J. A. Ruiz la define, aproximándose al primer camino señalado por Habermas, como: «la opinión de todos (mejor, del pueblo activo, del público participante) sobre los asuntos que afectan a todos. O de otro modo: la opinión del pueblo sobre lo público. Insistiendo en que el pueblo que genera la opinión pública es un pueblo activo, participante, que se aleja del pueblo masa, un pueblo al que se viene denominando público» (Ruiz, 1997: 39).

Uno de los problemas centrales a la hora de hablar de opinión pública es el de establecer el valor que se da a las distintas opiniones que la conforman. Una solución es proceder igualando los pesos de las opiniones dadas, como hace el modelo clásico de opinión pública, que, como nos recuerda Miguel Beltrán, implica lo siguiente: «Los investigadores de opinión parten de la premisa básica de la democracia de sufragio universal: "un ciudadano, un voto, un valor", igualan los votos con las expresiones de la opinión, y dan el mismo valor numérico a cada una de tales expresiones [...] La suma total de expresiones es presentada como una estimación de la "opinión pública" acerca de la cuestión

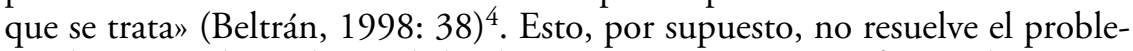
ma de cómo valorar el peso de las distintas opiniones que conforman la opinión pública.

En cuanto a la definición de nuevas biotecnologías, no existe un acuerdo unánime sobre cuáles son las tecnologías que se deben englobar en las mismas, y ni siquiera sobre la amplitud de campos científicos y tecnológicos que deben tener. En tal sentido, la: «biotecnología es como un concepto paraguas bajo el cual se agrupan técnicas y aplicaciones que cubren diversos campos [...] Incluso hoy en día muchas reuniones científicas sobre biotecnología empiezan con largos debates a propósito de la definición adecuada de biotecnología. Esta situación viene ejemplificada por la coexistencia de 41 definiciones diferentes de la biotecnología en los documentos de la Unión Europea» (Lemkow, 2002: 182). Además, tampoco hay acuerdo entre las técnicas, los tipos de investigación fundamental y las aplicaciones que estas nuevas tecnologías de la vida

4. Extraido de RokKan, S. «Comparative Cross-National Research». En: MERRITT, R. L.; RoKKan, S. (eds.). Comparing Nations. New Haven: Yale University Press. 
engloban. No obstante, en este artículo he optado por una definición de las mismas práctica, y que remite a que las nuevas biotecnologías son las que utilizan "comercialmente las técnicas de ADN recombinante, la fusión celular y los nuevos procedimientos de la bioingeniería» (Muñoz, 1995: 1), lo cual no quiere decir que en el análisis que efectúo sobre la opinión de los españoles de las nuevas biotecnologías, objeto de este artículo, no atienda a las valoraciones de tipo no económico: éticas, de riesgo para el medio ambiente, culturales, sociales, etc. Éstas son indudablemente muy importantes a la hora de establecer la aceptación de estas tecnologías, aceptación que es fundamental para el desarrollo que las mismas vayan a tener.

\section{La opinión de los españoles ante las nuevas biotecnologías}

Para establecer la opinión de los españoles ante las nuevas biotecnologías he tenido en cuenta cuatro estudios elaborados en la década de 1990. El primero, el de L. Moreno, L. Lemkow y A. Lizón: Biotecnología y sociedad. Percepción y actitudes públicas, fue publicado en 1992 por el Ministerio de Obras Públicas y Transportes español. Este estudio aunó metodologías cualitativas (grupos de discusión y talleres de trabajo) con cuantitativas (una encuesta, con el correspondiente análisis de los datos obtenidos a través de ella). El segundo estudio fue dirigido por Fabio Terragni, financiado por la Comisión de las Comunidades Europeas, que publicó sus resultados en 1995, su título fue: The public debate on biotechnology in Southern European Countries. De él tengo en cuenta las entrevistas realizadas en España ${ }^{5}$. El tercer estudio visto ha sido el de J. Atienza y J. L. Luján: La imagen social de las nuevas biotecnologias en España, publicado en 1997 por el Centro de Investigaciones Sociológicas (CIS). El método utilizado en él fue el de la encuesta y el análisis de datos de la misma. El último estudio analizado ha sido el del International Research Associates: The Europeans and biotechnology, publicado por la Comisión de las Comunidades Europeas en 2000. Su metodología fue la encuesta, y su correspondiente análisis, para los países de la Unión Europea.

A través de los resultados obtenidos en los estudios señalados ${ }^{6}$ me ha sido posible precisar, aunque de forma aproximativa y general, la percepción, la opinión y la actitud de los españoles ante las nuevas biotecnologías en la década de los noventa, y, a través de otras investigaciones realizadas en otros países, comparar si en las mismas existían o no diferencias, y cual era su sentido. Y ello a pesar de que, en las investigaciones analizadas en el caso español, no existe más que una coincidencia parcial en los temas tratados, y la dificultad comparativa que supone la gran variedad de metodologías usadas en las investigaciones consideradas: cualitativas, como en los grupos de discusión, talleres de

5. Buena parte de estas entrevistas fue realizada y trascrita por el autor de este artículo durante 1994.

6. Los resultados concretos de cada estudio los podemos encontrar en cada uno de ellos, pero también en su conjunto y de forma amplia en J. Taguenca (2003). 
trabajo, entrevistas en profundidad; y cuantitativas, como en las investigaciones basadas en cuestionarios. Esta variedad metodológica, a pesar de las dificultades de análisis que conlleva, da riqueza, a mi entender, a los resultados globales que presento en este artículo.

\subsection{Resultados obtenidos}

Una opinión informada sobre los distintos aspectos de la biotecnología necesita de una comprensión básica de las técnicas utilizadas, y de las ventajas y los riesgos que entrañan. Esta comprensión puede modificar la perspectiva de quien la alcanza. De hecho, dos estudios realizados en Estados Unidos por J. Doble y J. Johnson, y en Nueva Zelanda por P. K. Couchman y K. FinkJensen, mostraron cómo una mejor comprensión de la ciencia y la tecnología da como resultado un cambio hacia una opinión más favorable ${ }^{7}$. Esto, sin embargo, debe ser matizado en un doble sentido: en que el cambio no sólo se produce por un mayor conocimiento científico, sino también por una mejor comprensión de los complejos aspectos normativos que intervienen, y lo que es más importante: un mayor conocimiento del tema no siempre conduce a un mayor apoyo de la biotecnología ${ }^{8}$. En este sentido, un estudio británico realizado en 1988 reveló que los encuestados más informados en biotecnología eran los que mostraban las opiniones más firmes, tanto las que eran favorables como las que eran contrarias a las mismas ${ }^{9}$. Por tanto, tal como nos recuerda Christine Deane: «aunque informar al público de los hechos sobre la tecnología influye en su opinión, el resultado puede no ser siempre favorable para la biotecnología. Las creencias fundamentales acerca de Dios y la naturaleza del universo tienen un efecto profundo en las actitudes de las personas ante la biotecnología» (Deane, 1998: 387-388).

Los cuatro estudios españoles que analizó (E1, E2, E3 y E4 a partir de ahora $)^{10}$ consideran el conocimiento sobre las nuevas biotecnologías como un factor importante de diferenciación entre opiniones, y una buena variable a la hora de observar la penetración de estas tecnologías en la sociedad en cuanto a su posible apoyo a las mismas, aunque no se engañan al respecto de considerar que existe una relación positiva entre mayor conocimiento y mayor apoyo.

El conocimiento que tienen los españoles de las nuevas biotecnologías es muy bajo (E1 y E4), uno de los menores si lo comparamos con el que poseen los habitantes de otros países de la UE. (E4); aunque éstas han sido incorpora-

7. Véase, al respecto, Doble y Johnson (1994), y también Couchman y Fink-Jensen (1990).

8. Cfr. Deane (1998: 387).

9. Nos referimos al estudio de Evans, G.; DURANT, J. «The relationship between knowledge and attitudes in tehe public understanding of sciences in Britain», que apareció en la revista Public Undestanding of Science, no 4, 1995, p. 57-74.

10. El orden corresponde a la antigüedad de los mismos, de manera que E1 fue el primero en publicarse y E4 el último en aparecer. Este orden es el mismo que mencionamos al principio del apartado 3, cuando explicamos dichos estudios brevemente. 
das a su horizonte de expectativas y algunas de sus aplicaciones concretas les son conocidas (E1). La información de que disponen los españoles sobre ellas son sólo sobre conceptos genéricos (E1); tienen poca sobre sus avances, y tienen bastante sobre sus aplicaciones (E1 y E4), lo cual indica que el interés de los españoles se centra más sobre las posibles aplicaciones biotecnológicas que sobre su funcionamiento interno. En realidad, al igual que ocurre en otros países, los ciudadanos de nuestro estado consideran a las nuevas biotecnologías como un importante instrumento que encierra posibilidades de mejorar sus condiciones de vida, al tiempo que temen los abusos que se puedan cometer con ellas ${ }^{11}$. De ahí, como decíamos, que su interés sea más pragmático que sustantivo.

Lo observado sobre el conocimiento y la información que tienen los españoles sobre las nuevas biotecnologías no contradice el gran interés que muestran por éstas $(\mathrm{E} 4)^{12}$. Quizá la causa de esto sea, como ya señaló L. Lemkow: «Las recientes investigaciones de las ciencias sociales indican que la población no está bien informada y carece de un conocimiento detallado sobre las nuevas biotecnologías y sus aplicaciones específicas [esto es precisamente lo que se observa en los datos de los estudios que he examinado]. Esta conclusión representa una sobresimplificación de una situación mucho más compleja y puede deberse [...]: a la confusión en cuanto a las definiciones y al hecho de que en algunas encuestas los cuestionarios sean demasiado generales» (Lemkow, 1993: 11).

En cuanto a la aceptación de las nuevas biotecnologías, los españoles son los que más las aceptan de los residentes de la Unión Europea (E4), lo cual constituye un resultado lógico de su percepción muy positiva respecto a ellas y el impacto que tendrán (E1, E2 y E4). Esto está un tanto en contradicción con la resistencia ética y el temor que les provocan: en cuanto a la manipulación genética de células humanas o embriones, aunque ésta sea con fines de investigación (E1 y E3), sólo se admite la que se realiza terapéuticamente para el propio embrión o enfermo (E1 y E4). En general, los españoles aceptan las aplicaciones biotecnológicas destinadas a la salud humana, el medio ambiente y la agricultura (E1, E3 y E4), y rechazan las dirigidas a la producción de alimentos y la eugenesia (E1 y E3). Destaca también la distinta consideración que tienen de la investigación básica, a la que apoyan sin restricciones (E1 y E2), de las aplicaciones industriales, a las que temen (E1). En realidad, lo que ocurre es que las actitudes son muy sensibles a los aspectos concretos de la biotecnología de que se trate, siendo más aceptadas las que afectan a las plantas que las que afectan a los animales, y éstas que las que afectan a los humanos ${ }^{13}$.

11. Cfr. Marlier (1992).

12. Aunque el interés sea una condición en cierta forma necesaria para el conocimiento, nunca lo es suficientemente. Nos referimos aquí al hecho de que el interés predispone, pero esta predisposición, si no se traduce en acción de estudio de un objeto determinado, no redunda en una mayor información de él, y por tanto no supone su mejor comprensión. En definitiva, interés y conocimiento se unen como condición, en cierto modo, necesaria, pero no como condición suficiente, lo que supone la eliminación de la contradicción que pueda haber entre gran interés sobre un tema determinado y poco conocimiento sobre el mismo.

13. Cfr. Espey (1993), y Couchman y Fink-Jensen (1990). 
Esto debe matizarse para el caso español: las nuevas biotecnologías relacionadas con animales son tan aceptadas como las referidas a las plantas ${ }^{14}$.

La gran aceptación de los españoles respecto a estas nuevas tecnologías de la vida no viene acompañada de una carta blanca a las mimas. Lejos de ello, los residentes en nuestro país demandan que éstas sean controladas, a fin de evitar las consecuencias negativas que puedan tener (E1 y E2), y de las que muestran ser conscientes (E1, E2 y E3). Al respecto, ya en los grupos de discusión de L. Moreno, L. Lemkow y A. Lizón (1992), en su trabajo de campo de 1989, mencionaban que dicho control debería servir para no permitir la experimentación que no tuviera como finalidad la prevención y el tratamiento de enfermedades, asegurar la privacidad de la información genética propia de cada individuo, permitir la preservación de la variedad genética humana y la integridad física de las personas y dar garantía sobre la igualdad de acceso a los nuevos usos médicos procedentes de las nuevas biotecnologías ${ }^{15}$. Estas demandas de control no son exclusivas, ni mucho menos, de los españoles. Por ejemplo, un estudio realizado en Estados Unidos sobre terapia génica dio como resultado que el $90 \%$ de los encuestados estaba a favor de ella, pero el $70 \%$ de los mismos creía que ésta debía ser objeto de una regulación estric$\mathrm{ta}^{16}$. En el ámbito europeo, un estudio realizado en 1991 resaltó que, si bien existen diferencias importantes en el ámbito nacional en cuanto al apoyo obtenido por las distintas aplicaciones de la biotecnología, el deseo de un control público de las mismas era elevado en todos los encuestados del conjunto de los países ${ }^{17}$. Por último, como señalan Alicia Durán y Jorge Riechmann: «El llamamiento a la cautela y al control de las actitudes científicas y tecnológicas en el campo de las tecnologías genéticas y biológicas era la conclusión más importante del estudio realizado en siete países de la CE: Alemania, Dinamarca, España, Francia, Gran Bretaña, Grecia e Italia» (Durán y Riechmann, 1998: 11).

En cuanto a cómo debe realizarse el control, los españoles piensan que debe hacerse sobre la base de la creación de normativas reguladoras de las aplicaciones (E1 y E2). Sin embargo, nada manifiestan sobre cuál debe ser su contenido (E1 y E2), y nada saben sobre las existentes (E2). Y ello a pesar de que, como señala Christine Deane: «El informar al público de la normativa y de las garantías contra la utilización indebida puede mitigar la preocupación pública sobre las nuevas biotecnologías e influir en el nivel de apoyo que obtienen» (Deane, 1998: 388). Pero esto no siempre es así, en Alemania, por ejemplo: «la normativa no ha logrado aumentar significativamente la aceptación de la tecnología genética en general» ${ }^{18}$ (Brüser, 1998: 379). Por otra parte, hay que subrayar

14. Véase Lizón (1996).

15. Observaciones extraídas de un borrador de análisis de estos grupos de discusión, anterior a la publicación de este estudio. Agradezco a sus autores que me facilitaran el mismo.

16. Ver, al respecto, Beyertz (1995).

17. Véase Marlier (1992).

18. Hay que recordar que, en este país, se produjo un intenso debate sobre las nuevas biotecnologías, y sobre las normativas que las regulan, en las décadas de 1980 y 1990. 
algo importante que también subyace cuando hablamos de la implementación normativa en las nuevas biotecnologías. Y es, como señala Elvira Durán, que: «La complejidad, tanto teórica como de realización técnica, de la investigación biotecnológica y de su aplicación médica pone de manifiesto que el mero control legal es insuficiente para garantizar la calidad ética. Es insuficiente porque el control legal siempre es un control externo que sólo llega a algunas cuestiones y deja un amplio campo al arbitrio de la persona o grupo que dirige la investigación. Y también porque, debido a la velocidad con que se producen los avances, las decisiones y las valoraciones jurídicas llegan, generalmente, cuando ya no hay rectificación posible de lo hecho" (Durán, 1998: 394).

Por otro lado, los españoles rechazan a los agentes y a las instituciones más directamente involucrados en el desarrollo de las nuevas biotecnologías: industria, científicos y Administración, para que tengan el control de las mismas (E1), pero tampoco manifiestan qué instituciones y organismos deben ser los que controlen las nuevas biotecnologías ni quiénes deben formar parte de ellos, ni tampoco como debe ser la normativa que las regule (E1 y E2). En este sentido, se alejan de lo que nos dice Christine Deane: «Los procedimientos de consulta y participación sociales en la elaboración de las normas relativas a la biotecnología estimulan una actitud más positiva hacia la misma» (Deane, 1998: 390). No obstante, como se señaló en un estudio realizado en el Reino Unido por Brian Wynne y otros, publicado en 1997: «The development of genetically modified foods appeared to be seen as lying outside people's control, with little sphere for public choice or intervention» (Wynne y otros, 1997: 13), lo que se aleja, sin duda, del ideal de consulta y participación señalado por Christine Deane, que daría como resultado una actitud más positiva hacia las nuevas biotecnologías. En España tampoco se cumple este ideal, sin embargo, los españoles tienen, como muestran los estudios que nos sirven de base, una actitud positiva hacia las nuevas biotecnologías.

Los españoles reclaman más participación social en las decisiones a tomar sobre las nuevas tecnologías de la vida (E1 y E2), y lo hacen sin tener una idea clara sobre los mecanismos que deben articularse para canalizarla y siendo pesimistas respecto a que ella sea posible, o, de conseguirse, sea eficaz a la hora de impedir las consecuencias negativas que de ellas deriven (E1 y E2). Tampoco quieren involucrarse personalmente en esta participación (E1 y E2). No obstante, existe evidencia empírica a favor de la tesis de que cuanto mayor es la separación entre el deseo de participación de los ciudadanos en el proceso de toma de decisiones y la falta de participación percibida, más probable es que la sociedad crea que la biotecnología acarrea más peligros que ventajas para la sociedad, con el consiguiente rechazo de la misma ${ }^{19}$. Sin embargo, en España no existe participación percibida, como muestran varios de los estudios que nos sirven de referente (E1 y E2), y las nuevas biotecnologías, pese al temor que despiertan, no son rechazadas (E1, E2, E3 y E4). 
Existe percepción del riesgo ${ }^{20}$ que entrañan las nuevas biotecnologías (E1 y E2), lo que tiene como consecuencia una posición realista respecto a éstas: los riesgos se contraponen a los grandes beneficios que de ellas se esperan (E1 y E2). Existe, además, la creencia generalizada de que los beneficios serán mayores que las pérdidas que puedan producir las consecuencias negativas de estas nuevas tecnologías de la vida (E1 y E2), lo que es justamente lo contrario de lo que creen los alemanes. Éstos ven a las nuevas biotecnologías como algo amenazador, no realizan de las mismas ninguna distinción entre las diferentes aplicaciones, y las rechazan. En ellos existe una falta de confianza en la genética que afecta a la aceptación de sus aplicaciones ${ }^{21}$.

Los españoles más críticos con las nuevas biotecnologías opinan también que el riesgo debe examinarse caso por caso, que debe existir participación social para su control y que la normativa a crear debe ser eficaz para prevenirlo (E1 y E2). Estos tres aspectos son considerados por estos sectores como básicos e imprescindibles para aceptar las nuevas biotecnologías (E1). En realidad, estas consideraciones corresponden en buena medida a reacciones que tienen su origen en los cuatro principios de racionalidad para actuar en condiciones de riesgo y/o incertidumbre, y que Antoni Doménech enuncia de la siguiente forma: «1) En condiciones de riesgo, es racional actuar minimizando el riesgo, o, lo que es lo mismo, maximizando la utilidad esperada. 1bis) Es racional evitar todo curso de acción cuyas consecuencias puedan asociarse a estados futuros del mundo con costes infinitos, siempre que esos estados tengan una probabilidad superior a cero. 2) En condiciones de incertidumbre, es racional actuar como si lo peor fuese a pasar y, en consecuencia, limitarse a maximizar la mínima utilidad (criterio maximin), es decir, escoger aquel curso de acción que lleva al resultado menos malo de todos los resultados malos posibles... 3) En situaciones de incertidumbre y ambivalencia tecnológica, es racional actuar minimizando el máximo arrepentimiento: tomar la decisión que menos podamos lamentar (criterio del arrepentimiento mínimax). 4) Cuando domina la incertidumbre, es racional seguir 2) o 3), según convenga: 2) cuando los mayores beneficios que prometen las diversas alternativas andan parejos y 3) cuando estos beneficios difieren apreciablemente; cuando domina el riesgo, seguir 1)»(Doménech, 1986: 25).

Los españoles no tienen muy claro quién debe decidir sobre los desarrollos que se hagan de estas tecnologías (E1, E2 y E3). Si bien en el estudio de J. Atienza y J. L. Luján (1997) se manifestaban a favor de los científicos y la sociedad civil, aunque en menor medida que los anteriores, en los grupos de discusión del estudio de L. Moreno, L. Lemkow y A. Lizón (1992), quizá porque en ellos se profundizó más sobre el tema, los científicos fueron rechaza-

20. Recordemos, como señala J. Beriain, que: «El riesgo es la “medida”, la determinación limitada del azar según la percepción social del riesgo, surge como el dispositivo de racionalización, de cuantificación de metrización del azar, de reducción de la indeterminación, como opuesto del apeiron ("lo indeterminado")» (Beriain, 1996: 9). La evaluación del riesgo se basa en esta cuantificación del azar de la que nos habla Beriain.

21. Cfr. Brüser (1998). 
dos como decisores de dichos desarrollos, dada su vinculación interesada en los mismos. Esta posición de rechazo hacia los científicos como decisores por parte de los sectores más críticos de las nuevas biotecnologías también se mostró en una encuesta canadiense, donde los grupos de protección del medio ambiente y las asociaciones de consumidores inspiraron un grado de confianza mucho mayor que los científicos, las empresas de biotecnología y las autoridades públicas de control. A los científicos se los situaba en la esfera de influencia de las empresas, de ahí que su opinión se considerara interesada ${ }^{22}$.

En estos grupos españoles más críticos respecto a las nuevas biotecnologías se daba la responsabilidad del desarrollo de las nuevas biotecnologías a organismos independientes, en los que la participación de la sociedad civil se consideraba importante, aunque nada manifestaron sobre la composición que debían tener los mismos ni sobre cómo debían funcionar, y la responsabilidad personal brilló por su ausencia (E1 y E2).

Los agentes en quienes confían los españoles como fuentes de información y formación de opinión son: los ecologistas, las organizaciones no gubernamentales y los movimientos sociales, las organizaciones de consumidores y la comunidad científica; los únicos que confían en la Iglesia son los creyentes (E2). Por otro lado, confían poco o nada en los medios de comunicación de masas, la Administración pública, los partidos políticos y la industria (E2). En el Reino Unido, en una encuesta que investigaba las actitudes sociales frente a los alimentos modificados genéticamente, y se indagaba sobre la credibilidad que tenían distintas fuentes de información para los encuestados, las fuentes que recibieron una mayor calificación fueron varios programas de televisión, las organizaciones de consumidores, algunos periódicos y las publicaciones científicas. La confianza en la información difundida por el sector alimentario y la Administración era considerablemente inferior ${ }^{23}$. En España, las informaciones dadas por los medios de comunicación (televisión y prensa en general) son consideradas como sensacionalistas y valorativas, no se confía en ellas (E1 y E2); la de revistas especializadas no se utiliza para informarse (E1 y E2). Tampoco, como ocurre en el Reino Unido, se confía mucho en la información proporcionada por las empresas involucradas en las nuevas tecnologías de la vida ni en la dada por la Administración (E1 y E2).

Los españoles desconocen las iniciativas públicas y privadas que se realizan en torno a las nuevas biotecnologías en nuestro país (E2), lo cual tiene como consecuencia la preocupación sobre nuestra dependencia de las multinacionales que las desarrollan y las comercializan (E1 y E2).

En cuanto a las variables sociodemográficas, el nivel de estudios se muestra como la variable más importante (E1, E3 y E4), de manera que, a mayor nivel de ellos, mayor aceptación de las nuevas biotecnologías (E1, E3 y E4), aunque a este grupo pertenecen también los sectores más críticos de las mismas

23. Cfr. Frewer y Shepard (1994). 
(E1). Esta misma tendencia se observa respecto a mayores ingresos (E4), mayor conocimiento (E3), grupos de edades más jóvenes (E1 y E4) y cuando el sexo es masculino (E1). Las creencias religiosas no son discriminatorias: creyentes y no creyentes aceptan o rechazan las nuevas biotecnologías por igual, eso sí, sobre la base de distintas razones (E1).

En realidad, y como conclusión general al análisis que aquí hemos realizado, las investigaciones muestran que, en la década de los noventa, todavía se estaba en las primeras fases de formación de actitudes y éstas no se encontraban fijadas en una gran proporción de la población. Pero, en ello, España no se diferencia del resto del mundo, pues varios estudios realizados en distintos países muestran que éstos, al igual que el nuestro, estaban, en la década referida, también en las primeras fases en esa formación de actitudes respecto a las nuevas biotecnologías ${ }^{24}$.

\section{Referencias bibliográficas}

ATIENZA, J.; LUjÁN, L. (1997). La imagen social de las nuevas biotecnologías en España. Madrid: Centro de Investigaciones Sociológicas.

BELTRÁN, M. (1998). «Cinco vías de acceso a la realidad social». En: GARCíA FERRANDO, Manuel; IBÁÑEZ, Jesús; Alvira, Francisco (eds.). El análisis de la realidad social. Métodos y técnicas de investigación. Madrid: Alianza Universidad, p. 19-49.

BERIAIN, J. (1996). «Prólogo». En: BERIAIN, Josetxo (comp.). Las consecuencias perversas de la modernidad. Barcelona: Anthropos.

BeyerTZ, K. (1995). "Ethics, genetic engineering and the public». Genetics, CIBA Comunications.

Bilbeny, N. (1992). Aproximación a la ética. Barcelona: Ariel.

BRÜSER, T. (1998). «Comentarios sobre la tecnología genética en alemania». En: Romero Casabona, Carlos María (ed.). Biotecnología y Derecho. Perspectiva en Derecho Comparado. Bilbao-Granada: Cátedra Interuniversitaria-Fundación BBVDiputación Foral de Bizcaia, p. 379-384.

Couchman, P. K.; FinK-Jensen, K. (1990). «Public attitudes to genetic engineering in New Zeland». DSIR Crop Research Repport, no 138, noviembre.

DEANE, C. (1998). «La percepción social de la biotecnología». En: Romero CASABONA, Carlos María (ed.). Biotecnología y Derecho. Perspectiva en Derecho Comparado. Bilbao-Granada: Cátedra Interuniversitaria-Fundación BBV- Diputación Foral de Bizcaia, p. 385-391.

Doble, J.; Johnson, J. (1994). Science and the Public. Nueva York: The Public Agenda Foundation.

DOMÉNECH, A. (1986). «La ciencia moderna, los peligros antropogénicos presentes y la racionalidad de la política de la ciencia y la técnica». Arbor, no 481, p. 9-51.

Durán, A.; RiechmanN, J. (1998). «Tecnologías genéticas y ética de la I+D». En: DuRÁn Alicia; RiechmanN, Jorge (coord.). Genes en el laboratorio y en la fábrica. Madrid: Editorial Trotta y Fundación $1^{\circ}$ de Mayo, p. 9-21.

DURÁN, E. (1998). «La reflexión ética ante el avance de la biotecnología». En: ROMERO Casabona, Carlos María (ed.). Biotecnología y Derecho. Perspectiva en Derecho 
Comparado. Bilbao-Granada: Cátedra Interuniversitaria-Fundación BBVDiputación Foral de Bizcaia, p. 393-398.

ESPEY, J. (1993). Report to Canadian Institute of Biotechnology on attitudes towards Biotechnology. Ontario.

Evans, G.; DuRANT, J. (1995). «The relationship between knowledge and attitudes in tehe public understanding of sciences in Britain». Public Understanding of Science, $\mathrm{n}^{\mathrm{o}}$ 4, p. $57-74$.

FREWER, L. J.; SHEPARD, R. (1994). «Attributing information to different sources. Effects on percieved qualities on information on the perceived relevance of information, and on attitude formation». Public Understanding of Science, no 3, p. 385-402.

HABERMAS, J. (1981). Historia y crítica de la opinión pública. La transformación estructural de la vida pública. Barcelona: Gustavo Gili.

INTERNATIONAL RESEARCH ASSOCIATES (2000). Eurobarometer 52.1. The europeans and biotechnology. Luxemburgo: Oficina de Publicaciones de las Comunidades Europeas, marzo.

KRIMSKY, S. (1992). «Regulating Recombinant DNA Research». En: NeLKIN, Dorothy (ed.). Controversy Politics of Technical Decisions. Londres: Sage Publications, p. 251-280.

LEMKOW, L. (1993). Actitudes públicas hacia la ingeniería genética: algunas perspectivas europeas. Luxemburgo: Oficina de Publicaciones Oficiales de las Comunidades Europeas.

LeMKow, L. (2002). Sociología ambiental. Pensamiento socioambiental y ecología social del riesgo. Barcelona: Icaria.

LIZON, A. (1996). A metric evaluation of public perception in the face of biotechnological risk. 4 vols. Informe del estudio CEC (BIO-CT94-0012).

MARLIER, E. (1992). «Eurobarometer 35.1, opinions of Europeans on biotechnology in 1991». Biotechnology in public, a review of recent research. Science Museum of the European Federation of Biotechnology.

Moreno, L.; Lemkow, L.; Lizón, A. (1992). Biotecnología y sociedad. Percepción y actitudes públicas. Madrid: Centro de Publicaciones del Ministerio de Obras Públicas y Transportes, Secretaría General Técnica.

MuÑoz, E. (1995). «Ingeniería genética en el sector primario y secundario: beneficios y problemas». Documento de trabajo 95-01 del Instituto de Estudios Sociales Avanzados. Madrid: Consejo Superior de Investigaciones Científicas.

REDCLIFT, M.; WOODGATE, G. (1997). «Sociology and environment discordant discourse». En: REDCLIFT, Michael; BENTON, Ted (eds.). Social Theory and the Global Environment. Londres y Nueva York: Routledge, p. 51-66.

RuIZ, J. A. (1997). Introducción a la tradición clásica de la opinión pública. Madrid: Tecnos.

Solleiro, J. L. (1990). «Patentes en biotecnología: oportunidades, amenazas y opciones para América Latina». Derecho Industrial, enero-abril. Buenos Aires, p. 107-135.

TAGUENCA, J. (2003). Las nuevas biotecnologias en España. Consideraciones sociológicas sobre sus politicas, controversias y opiniones. Tesis doctoral. Universidad Autónoma de Barcelona.

TerRagni, F. (1995). The public debate on biotechnology in Southern European Countries. Investigación financiada por la Comisión de las Comunidades Europeas (Contrato BIO2-CT93-603).

Wynne, B. y otros (1997). Uncertain World. Genetically Modified Organisms, Food and Public Attitudes in Britain. Lancaster: Lancaster University. 